\title{
miR-214 suppresses the osteogenic differentiation of bone marrow-derived mesenchymal stem cells and these effects are mediated through the inhibition of the JNK and p38 pathways
}

\author{
YONGZHI GUO, LIANHUA LI, JIE GAO, XIAOBIN CHEN and QINGHUA SANG \\ Department of Orthopedics, Beijing Army General Hospital, Dongcheng, Beijing 100700, P.R. China
}

Received April 18, 2016; Accepted November 30, 2016

DOI: $10.3892 / \mathrm{ijmm} .2016 .2826$

\begin{abstract}
In this study, we sought to investigate the expression of microRNA (miR)-214 on the osteogenic differentiation of bone marrow-derived mesenchymal stem cells (BMSCs) and explore the possible underlying mechanisms. We found that the overexpression of miR-214 effectively promoted the adipocyte differentiation of BMSCs in vitro, reduced alkaline phosphatase (ALP) activity and the gene expression of collagen type I (Col I), osteocalcin (OCN) and osteopontin (OPN) in the BMSCs. We further found that the overexpression of miR-214 suppressed the protein expression of fibroblast growth factor (FGF), phosphorylated c-Jun N-terminal kinase (p-JNK) and phosphorylated p38 (p-p38) in the BMSCs. The downregulation of miR-214 promoted the osteogenic differentiation of BMSCs, and increased ALP activity and Col I, OCN and OPN gene expression in the BMSCs. It also increased FGF p-JNK and p-p38 protein expression in the BMSCs. The use of JNK inhibitor (SP600125) enhanced the inhibitory effects of miR-214 overexpression on osteogenic differentiation, ALP activity, and $\mathrm{Col}$ I, OCN and OPN gene expression in the BMSCs. Lastly, the use of p38 inhibitor (SB202190) also enhanced the inhibitory effects of miR-214 overexpression on ALP activity, and Col I, OCN and OPN gene expression in the BMSCs. These results provide a mechanism responsible for the suppressive effects of miR-214 on the osteogenic differentiation of BMSCs involving the inhibition of the JNK and p38 pathways.
\end{abstract}

\section{Introduction}

Chronic inflammatory bone disease has the major characteristics of osteopenia, mainly including post-menopausal

Correspondence to: Dr Yongzhi Guo, Department of Orthopedics, Beijing Army General Hospital, 5 Nanmencang Road, Dongcheng, Beijing 100700, P.R. China

E-mail: yongzhiguopw@163.com

${ }^{*}$ Contributed equally

Key words: microRNA-214, bone marrow-derived mesenchymal stem cells, c-Jun N-terminal kinase, p38 osteoporosis, rheumatoid arthritis and periodontal disease. The disease is widely observed on a global scale, and is the primary cause of fractures and bone loss, and seriously threatens human health (1). The suppression of bone-forming ability is the main pathogenesis of the disease. Methods with which to enhance bone-forming ability are of major importance in providing a cure for the disease. Bone marrow-derived mesenchymal stem cells (BMSCs) are adult stem cells with self-renewal and multidirectional differentiation ability. Under different induction environments, they can differentiate into osteoblasts, adipocytes, chondroblasts and neuroblasts (2). Studies have indicated that BMSCs are the source of osteoblasts and osteocytes $(3,4)$. Inducing BMSCs to differentiate directionally is of significance to curing inflammatory bone disease and repairing bone loss $(3,4)$. However, the specific mechanisms involved remain unclear, as osteogenic differentiation involves a complex network, including the regulation of multiple signaling pathways at the transcriptional and post-transcriptional level (3).

MicroRNAs (miRNAs or miRs) are non-coding RNAs approximately 18-25 nucleotides in length and have been discovered in recent years. By targeting the 3'UTR of target genes, they can restrain the translation and stability of genes (5). It has been demonstrated that miRNAs plays an important role in regulating the self-renewal and differentiation ability of stem cells (6). Studies have found that miRNAs exert a regulatory effect on osteogenic differentiation in different cells; however, the osteogenic differentiation of stem cells is an extremely complex process and may involve the mutual coordination of multiple factors and different signaling pathways on different levels $(6,7)$. As a result, studying the regulatory role of miRNAs in the osteogenic differentiation of BMSCs may be crucial to providing a cure for osteoporosis and bone loss.

As adult stem cells derived from mesoblast, BMSCs can not only differentiate into adult cells from mesoblasts, including osteocytes, chondrocytes and adipocytes under the function of an appropriate inducer for culture in vitro, but can also can transdifferentiate into nerve cells from the neuroderm (8). Therefore, in the process of transdifferentiation, it is necessary to transmit the signal with induced differentiation into cells through the signal transduction pathway, so as to induce cell differentiation and impact outcomes. Mitogen-activated protein kinase (MAPK) is a type of serine/threonine protein kinase in 
cells. When extracellular growth factors are combined with corresponding receptors of the cytomembrane, this activates the MAPK cascade reaction, so as to impact the expression of corresponding cellular transcription factors and impact cell proliferation, differentiation and outcomes $(9,10)$.

With the development of cytobiology, it has been realized that cell adhesion is not a pure mechanical connection. Instead, it also involves complex signal transduction pathways (8). When studying the categories, structure and functions of the cell surface for adhesion molecules, the investigation of the signal transduction process mediated by these adhesion molecules has already become the hotspot in the field of life science. In a previous study, it was demostrated that genistein promotes osteoblastic differentiation through the p38 mitogen activated protein kinase (MAPK)-core-binding factor 1 (Cbfa1) pathway. Downstream JUN/p38 is activated (9). Thus, JUN/p38 may participate in the process of cell differentiation and proliferation. However, the mechanisms and function of miR-214 in the osteogenic differentiation of BMSCs remain unclear. Thus, the purpose of the present study was to investigate the function and possible molecular mechanisms of miR-214 in the osteogenic differentiation of BMSCs.

\section{Materials and methods}

Animals, isolation of BMSCs and cell culture. Male SpragueDawley rats ( $\mathrm{n}=6$; weighing, 180-210 g) were purchased from Beijing Vital River (Beijing, China). This study was approved by the Ethics Committee of Beijing Army General Hospital, Beijing, China.The rats were then sacrificed by decollation under $5 \%$ chloral hydrate and sterilized using $75 \%$ ethyl alcohol for 3-5 min. The femur of each rat was separated and excess tissue was removed using surgical scissors. The bone marrow was flushed out using Dulbeccos modified Eagle's medium (DMEM; HyClone, Waltham, MA, USA) and the bone marrow cells were washed and filtered using a 200 mesh sieve. Bone marrow cell were collected following centrifugation at $500 \mathrm{x} \mathrm{g}$ for $10 \mathrm{~min}$. The cells were washed with PBS and cultivated with red blood cell lysis buffer. The cell precipitate $\left(1-2 \times 10^{6}\right)$ was cultivated with DMEM supplemented with $10 \%$ fetal bovine serum (FBS), $100 \mathrm{U} / \mathrm{ml}$ penicillin and $100 \mathrm{lg} / \mathrm{ml}$ streptomycin) (all from HyClone) and seeded in a 6 well-plate at $37^{\circ} \mathrm{C}$ in $5 \% \mathrm{CO}_{2}$. The culture medium was replaced every 3 days. Primary rat BMSCs were digested with $10 \%$ trypsin after large colonies had formed and became confluent.

Transfection of cells with miR-214 and anti-miR-214 plasmids. miR-214 and the negative control plasmids, and the anti-miR-214 plasmid were packaged using lentiviral vectors generated by GeneChem (Shanghai, China). Lentivirus (1x10 $7 \mathrm{TU} / \mathrm{ml}$; Invitrogen, Carlsbad, CA, USA) was used to infect the cells according to the manufacturer' s instructions. Untransfected cells were used as controls. The cells were then cultured in fresh medium for further experiments after the lentivirus for $8 \mathrm{~h}$.

Osteogenic differentiation of BMSCs, treatment with p38 and c-Jun N-terminal kinase (JNK) inhibitors and Oil Red $O$ staining. Osteogenic differentiation was induced using standard osteoblast induction medium $\left(10^{-8} \mathrm{M}\right.$ dexamethasone, $0.2 \mathrm{mM}$ l-ascorbic acid, $10 \mathrm{mM}, \beta$-glycerophosphate and $10 \mathrm{mM}$ 1.25-vitamin D3), as previously described (11). The JNK inhibitor, SP600125 (final concentration $20 \mu \mathrm{M}$; MedChemexpress, Princeton, NJ, USA), and the p38 inhibitor, SB202190 (final concentration $20 \mu \mathrm{M}$; MedChemexpress), were added to the BMSCs for 7 days. The BMSCs were fixed in $10 \%$ formalin for $30 \mathrm{~min}$ at room temperature and stained with Oil Red O (Beyotime, Jiangsu, China) to assess adipocyte differentiation. Subsequently, $85 \%$ propylene glycol was added to the cells for $5 \mathrm{~min}$ and the cells were observed under a microscope (LSM510 Meta Confocal Microscope; Carl Zeiss, Oberkochen, Germany).

Alkaline phosphatase (ALP) activity assay. The BMSCs transfected with the miR-214 or anti-miR-214 plasmids, or treated with JNK inhibitor or p38 inhibitor were seeded at a density of $2 \times 10^{5}$ cells/well in 24-well plates and subjected to osteogenic differentiation. The BMSCs were lysed with RIPA lysis buffer (Thermo Fisher Scientific, Wilmington, DE, USA) supplemented with protease inhibitor. The protein concentration was determined by Bradford assay (Bio-Rad, Hercules, CA, USA) on a microplate spectrophotometer (Tecan, Port Melbourne, Australia). ALP activity was determined using an ALP activity kit (Nanjing Jiancheng Biotech, China) on a microplate spectrophotometer (Tecan).

Reverse transcription-quantitative PCR (RT-qPCR). Total RNA was extracted from the BMSCs transfected with miR-214 or anti-miR-214 plasmids, or treated with JNK inhibitor or p38 inhibitor using TRIzol reagent (Takara, Dalian, China). A total of $2 \mu \mathrm{l}$ of total RNA was reverse transcribed into cDNA using a HiFi-MMLV cDNA kit (Tiangen, Beijing, China). The Quant SYBR-Green PCR kit (Bio-Rad, Munich, Germany) was used for qPCR. The temperature profile of the reaction was $95^{\circ} \mathrm{C}$ for $2 \mathrm{~min}$, followed by 40 cycles of denaturation at $95^{\circ} \mathrm{C}$ for $20 \mathrm{sec}$, annealing at $60^{\circ} \mathrm{C}$ for $30 \mathrm{sec}$ and an extension at $72^{\circ} \mathrm{C}$ for $45 \mathrm{sec}$. The relative quantitation was calculated using the $2^{-\Delta \Delta \mathrm{Ct}}$ method. The sequences of the primers are listed in Table I.

Western blot analysis. The BMSCs transfected with miR-214 or anti-miR-214 plasmids, or treated with JNK inhibitor or p38 inhibitor were lysed on ice for $30 \mathrm{~min}$ in RIPA lysis buffer (Thermo Fisher Scientific) supplemented with protease inhibitor. The protein concentration was determined by Bradford assay (Bio-Rad) on a microplate spectrophotometer (Tecan). A total of $40 \mu \mathrm{g}$ proteins was resolved on a $10 \%$ sodium dodecyl sulfate-polyacrylamide gel electrophoresis (SDS-PAGE) gel and electro-transferred onto polyvinylidene difluoride (PVDF) membranes (Pall Corporation, New York, NY, USA). The membranes were incubated with the appropriate primary antibodies [anti-fibroblast growth factor (FGF; 1:1,000; 9740), anti-phosphorylated (p-)JNK (1:2,000; 4668), antip-p38 (1:2,000; 4511) (all from Cell Signaling Technology, Danvers, MA, USA) and GAPDH (1:5,000; H00002597-D01P; Abnova, Taiwan, China) at $4^{\circ} \mathrm{C}$ overnight. The membranes were then incubated with an HRP-conjugated secondary antibody (Xi'an Kehao Biological Engineering Co. Ltd, Xi'an, China) and developed by enhanced chemiluminescence (ECL; Millipore, Billerica, MA, USA). Protein expression was analyzed using an Odyssey Two-Color Infrared Imaging System (LI-COR Biosciences, Lincoln, NE, USA). 
Table I. Primer sequences use for PCR.

\begin{tabular}{lll}
\hline Gene & \multicolumn{1}{c}{ Forward primer } & \multicolumn{1}{c}{ Reverse primer } \\
\hline miR-214 & 5'-AGCCGACAGCAGGCACAGACA-3' & 5'-AGCCGACAGCAGGCACAGACA-3' \\
U6 & 5'-CGCTTCACGAATTTGCGTGTCAT-3 & 5'-GCTTCGGCAGCACATATACTAAAAT-3 \\
Col I & 5'-CTGCCCAGAAGAATATGTATCACC-3' & 5'-GAAGCAAAGTTTCCTCCAAGACC-3' \\
OCN & 5'-GAGGGCAATAAGGTAGTGAA-3' & 5'-CATAGATGCGTTTGTAGGC-3' \\
OPN & 5'-CCAAGCGTGGAAACACACAGCC-3' & 5'-GGCTTTGGAACTCGCCTGACTG-3' \\
Actin & 5'-GTAAAGACCTCTATGCCAACA-3' & 5'-GGACTCATCGTACTCCTGCT-3' \\
\hline
\end{tabular}

Col I, collagen type I; OCN, osteocalcin; OPN, osteopontin.

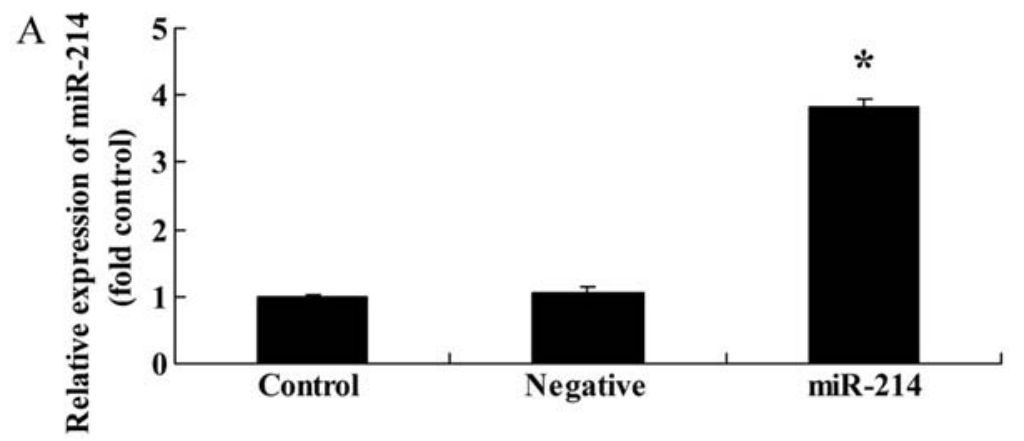

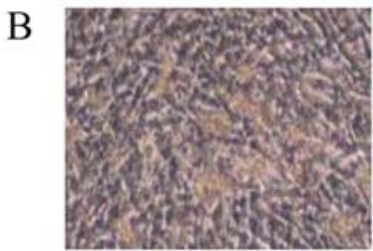

Control

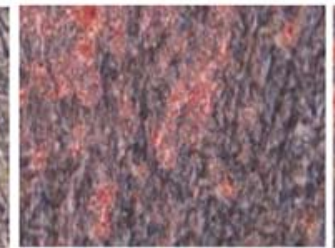

Negative

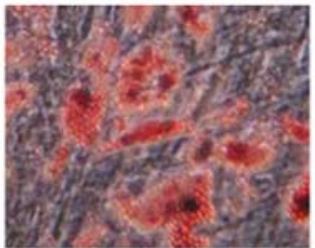

miR-214

Figure 1. Overexpression of miR-214 promotes the adipocyte differentiation of bone marrow-derived mesenchymal stem cells (BMSCs). (A) miR-214 expression increased following transfection with miR-214 overexpression plasmid; (B) overexpression of miR-214 promoted the adipocyte differentiation of BMSCs, as shown by Oil Red O staining; magnification, $\mathrm{x} 400$. Control, control group; negative, negative infection group; miR-124, miR-124 infection group. * $<<0.01$ compared with the control group.

Statistical analysis. All measurements are expressed as the means \pm SD. Comparisons were made using one/two-way analysis of variance (ANOVA) followed by the Turkey post hoc test for multiple comparisons. A value of $\mathrm{P}<0.05$ was considered to indicate a statistically significant difference.

\section{Results}

Overexpression of $m i R-214$ promotes the adipocyte differentiation of BMSCs. To investigate the miRNAs involved in the differentiation of BMSCs, an miR-214 overexpresion plasmid was constructed and transfrected into the BMSCs. Transfection with miR-214 plasmid significantly increased miR-214 expression in the BMSCs (Fig. 1A) and promoted the adipocyte differentiation of BMSCs (Fig. 1B).

Overexpression of miR-214 suppresses ALP activity and collagen type I (Col I), osteocalcin (OCN) and osteopontin (OPN) gene expression in BMSCs. We then examined the effects of the overexpression of miR-214 on ALP activity and Col I, OCN and OPN gene expression in BMSCs. We performed ALP activity assay and RT-qPCR. As expected, the overexpression of miR-214 significantly suppressed ALP activity and inhibited Col I, OCN and OPN gene expression in the BMSCs (Fig. 2). These results indicated that the overexpression of miR-214 suppressed the osteogenic differentiation of BMSCs.

Overexpression of $\mathrm{miR}-214$ suppresses $F G F$ protein expression in BMSCs. To determine whether FGF is a direct target of miR-214 in BMSCs, we measured FGF protein expression in the BMSCs following the overexpression of miR-214. As shown in Fig. 3, there was a significant inhibition of FGF protein expression in the BMSCs following the overexpression of miR-214.

Overexpression of miR-214 suppresses $p$-JNK protein expression in BMSCs. In addition, western blot analysis was applied to examine the effects of miR-214 on JNK protein expression. Compared with the controls, the overexpression of miR-214 significantly suppressed $\mathrm{p}-\mathrm{JNK}$ protein expression in the BMSCs (Fig. 4). 
A

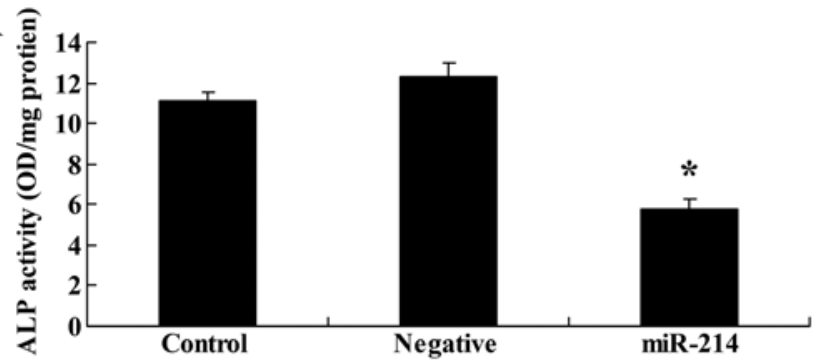

č

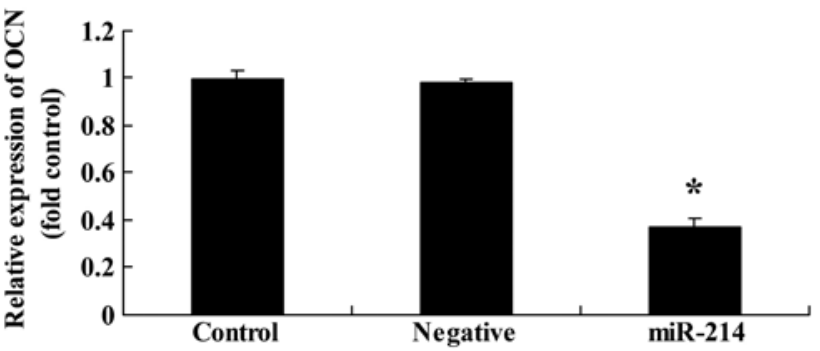

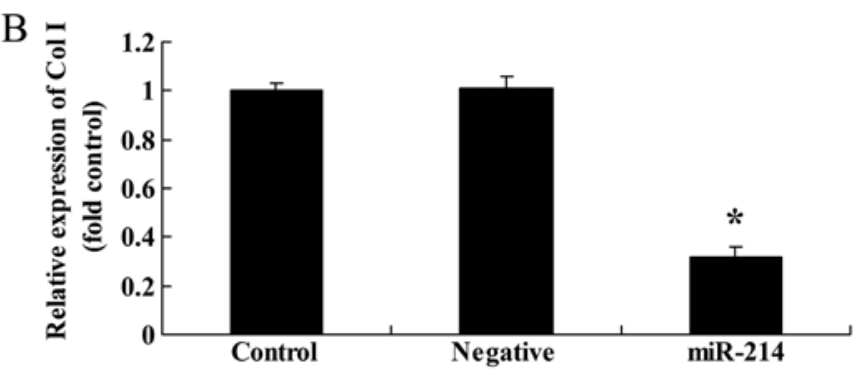

$\mathrm{D}$

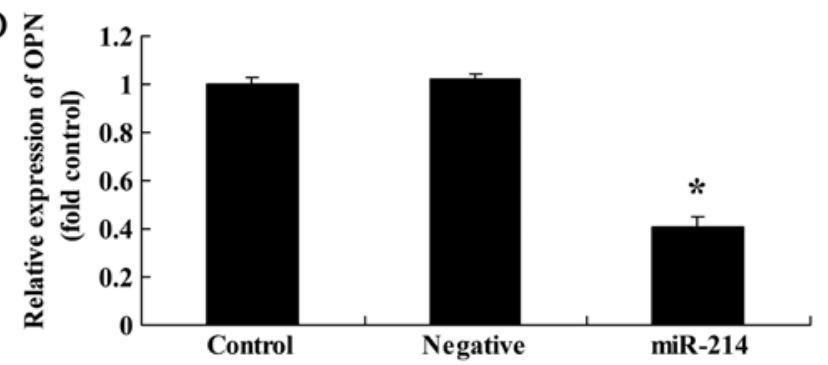

Figure 2. Overexpression of miR-214 suppresses alkaline phosphatase (ALP) activity, and decreases collagen type I (Col I), osteocalcin (OCN) and osteopontin (OPN) gene expression in bone marrow-derived mesenchymal stem cells (BMSCs). (A) Overexpression of miR-214 suppressed ALP activity and decreased (B) Col I, (C) OCN and (D) OPN gene expression in BMSCs. Control, control group; negative, negative infection group; miR-124, miR-124 infection group. ${ }^{*} \mathrm{p}<0.01$ compared with the control group.
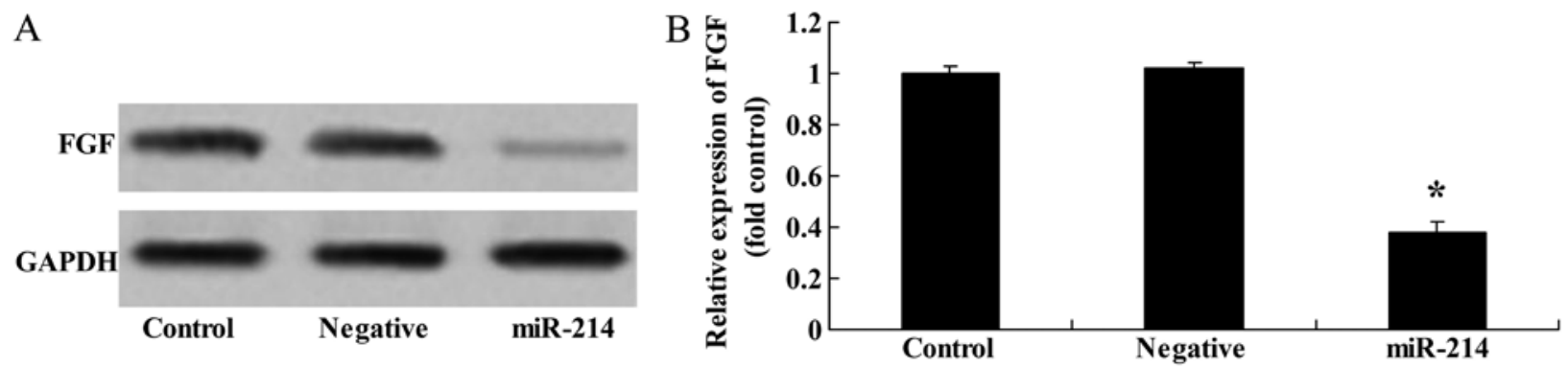

Figure 3. Overexpression of miR-214 suppresses fibroblast growth factor (FGF) protein expression in bone marrow-derived mesenchymal stem cells(BMSCs). Overexpression of miR-214 suppressed FGF protein expression as shown by (A) western blot analysis and (B) statistical analysis of FGF protein expression in BMSCs. Control, control group; negative, negative infection group; miR-124, miR-124 infection group. * $\mathrm{p}<0.01$ compared with the control group.

Overexpression of miR-214 suppresses p-p38 protein expression in BMSCs. To determine whether the overexpression of miR-214 affects p38 protein expression in BMSCs, western blot analysis was then performed to examine the effects of the overexpression of miR-214 expression on p-p38 protein expression in BMSCs. The overexpression of miR-214 significantly inhibited p-p38 protein expression in the BMSCs (Fig. 5).

Downregulation of miR-214 promotes the osteogenic differentiation of BMSCs. Conversely, we examined whether the downregulation of miR-214 affects the osteogenic differentiation of BMSCs. Anti-miR-214 plasmid was tansfected into the BMSCs. We found that the inhibition of miR-214 significantly decreased miR-214 expression in the BMSCs (Fig. 6A) and promoted the osteogenic differentiation of BMSCs (Fig. 6B).

Downregulation of miR-214 enhances ALP activity, and increases Col I, OCN and OPN gene expression in BMSCs. We then examined whether the downregulation of miR-214 also affects ALP activity, and Col I, OCN and OPN gene expression in BMSCs. The downregulation of miR-214 significantly increased ALP activity and promoted Col I, OCN and OPN gene expression in the BMSCs (Fig. 7).

Downregulation of $m i R-214$ increases FGF protein expression in BMSCs. To further determine whether FGF is a direct target of miR-214 in BMSCs, we measured FGF protein expression in BMSCs following the downregulation of miR-214. There was a significant increase in FGF protein expression in the BMSCs following the downregulation of miR-214 (Fig. 8).

Downregulation of miR-214 increases $p$-JNK protein expression in BMSCs. When miR-214 expression was downregulated, western blot analysis was applied to examine the effects of miR-214 inhibition on JNK protein expression. The downregulation of miR-214 significantly increased $\mathrm{p}-\mathrm{JNK}$ protein expression in BMSCs (Fig. 9).

Downregulation of miR-214 increases p-p38 protein expression in BMSCs. To further determine whether the downregulation of miR-214 affects p38 protein expression in BMSCs, western blot analysis was then performed to examine the effects of 
A
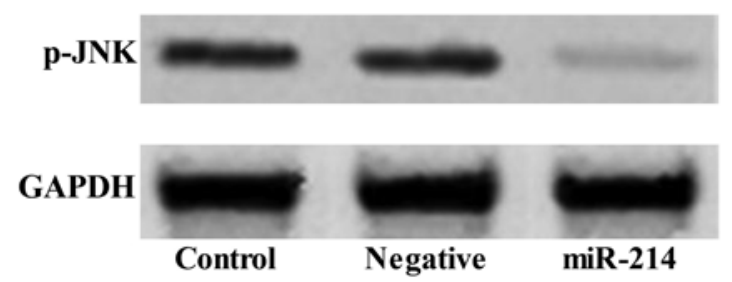
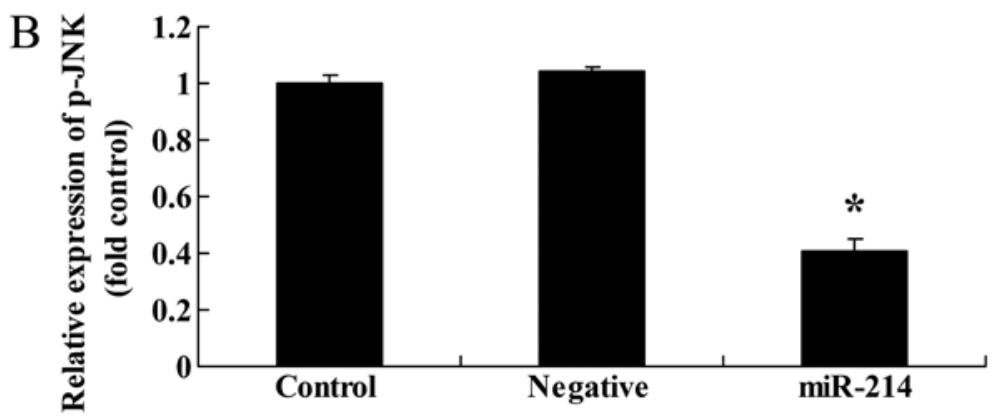

Figure 4. Overexpression of miR-214 suppresses p-c-Jun N-terminal kinase (JNK) protein expression in bone marrow-derived mesenchymal stem cells (BMSCs). Overexpression of miR-214 suppressed p-JNK protein expression as shown by (A) western blot analysis and (B) statistical analysis of p-JNK protein expression in BMSCs. Control, control group; negative, negative infection group; miR-124, miR-124 infection group. " p $<0.01$ compared with the control group.
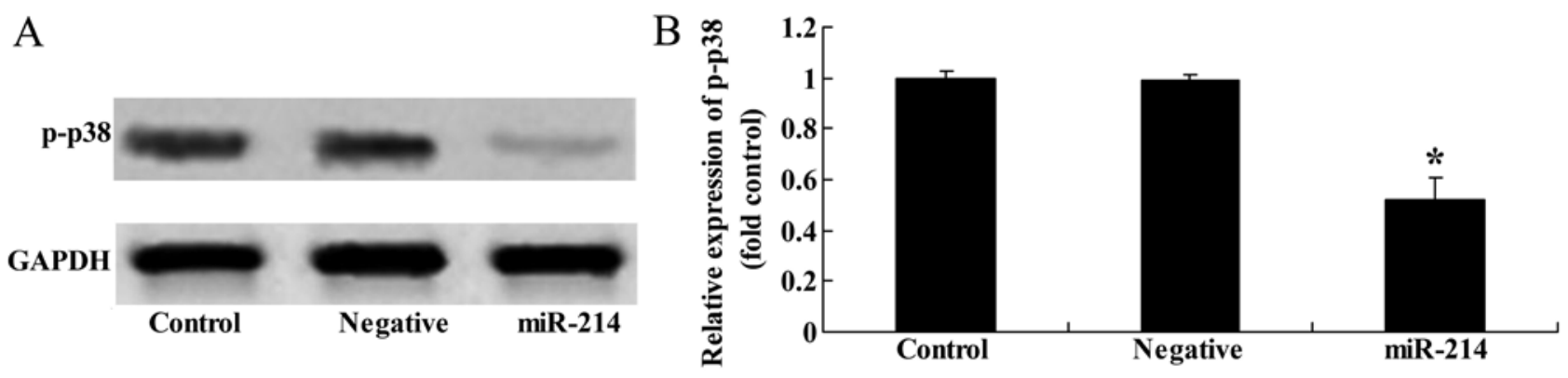

Figure 5. Overexpression of miR-214 suppresses p-p38 protein expression in bone marrow-derived mesenchymal stem cells (BMSCs). Overexpression of miR-214 suppressed p-p38 protein expression as shown by (A) western blot analysis and (B) statistical analysis of p-p38 protein expression in BMSCs. Control, control group; negative, negative infection group; miR-124, miR-124 infection group. ${ }^{*} \mathrm{p}<0.01$ compare $\mathrm{d}$ with the control group.

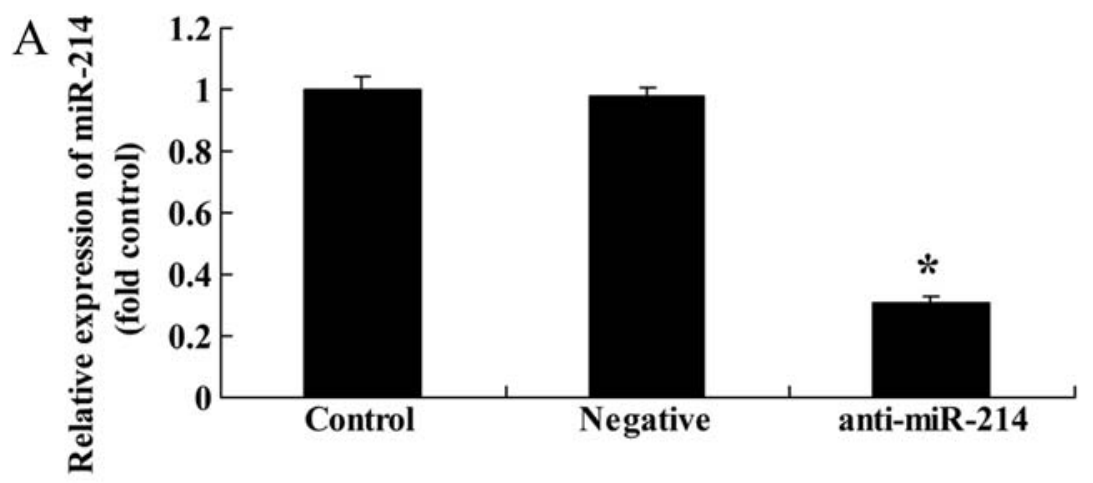

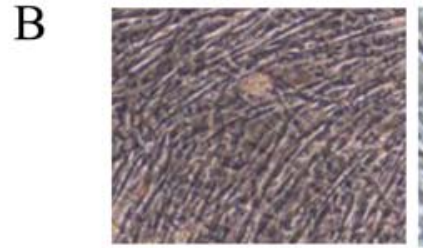

Control

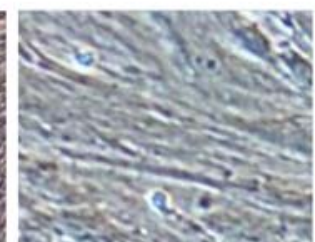

Negative

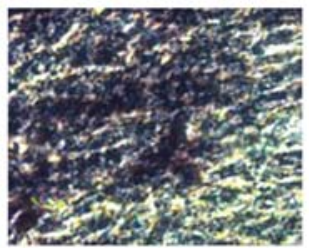

anti-miR-214

Figure 6. Downregulation of miR-214 promotes the osteogenic differentiation of bone marrow-derived mesenchymal stem cells (BMSCs). (A) miR-214 expression was downregulated by transfection with anti-miR-214 plasmid. (B) The downregulation of miR-214 increased the osteogenic differentiation of BMSCs. Control, control group; negative, negative infection group; anti-miR-124, anti-miR-124 infection group. * $\mathrm{p}<0.01$ compared with the control group.

the downregulation of miR-214 expression on $\mathrm{p}$-p38 protein expression in BMSCs. p-p38 protein expression in the BMSCs was significantly increased by the downregulation of miR-214 (Fig. 10).
Effect of the inhibition of JNK expression on the adipocyte differentiation of BMSCs following the overexpression of $m i R-214$. To assess the significance of the inhibition of JNK expression on the osteogenic differentiation of BMSCs 

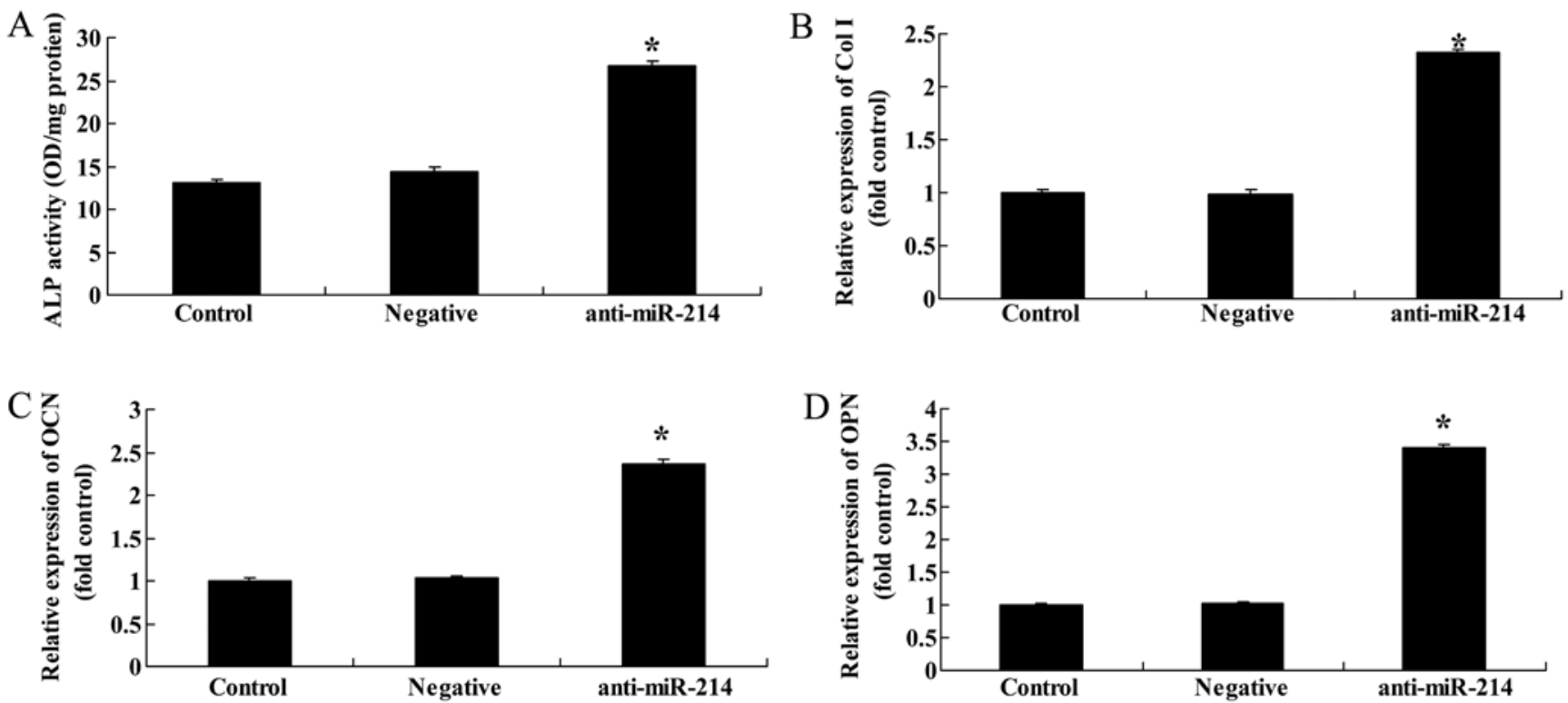

Figure 7. Downregulation of miR-214 activates alkaline phosphatase (ALP) activity and collagen type I (Col I), osteocalcin (OCN) and osteopotin (OPN) gene expression in bone marrow-derived mesenchymal stem cells (BMSCs). Downregulation of miR-214 suppressed (A) ALP activity, and decreased (B) Col I, (C) OCN and (D) OPN gene expression in BMSCs. Control, control group; negative, negative infection group; anti-miR-124, anti-miR-124 infection group. ${ }^{*} \mathrm{p}<0.01$ compared with the control group.

A

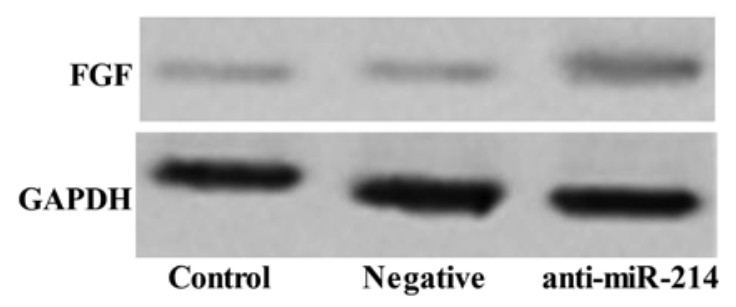

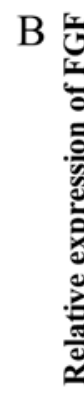

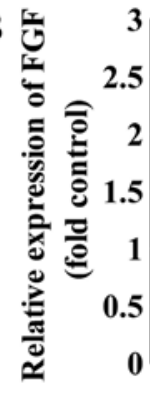

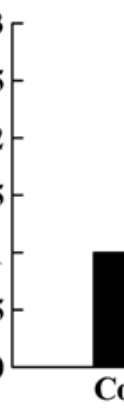

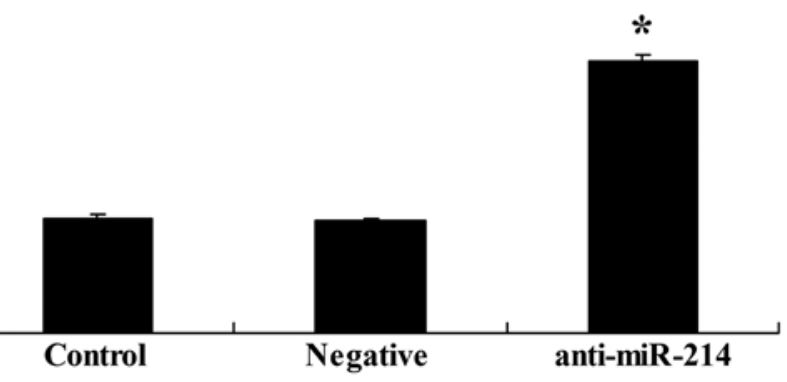

Figure 8. Downregulation of miR-214 promotes fibroblast growth factror (FGF) protein expression in bone marrow-derived mesenchymal stem cells (BMSCs). Downregulation of miR-214 suppressed FGF protein expression as shown by (A) western blot analysis and (B) statistical analysis of FGF protein expression in BMSCs. Control, control group; negative, negative infection group; anti-miR-124, anti-miR-124 infection group. *p<0.01 compared with the control group.
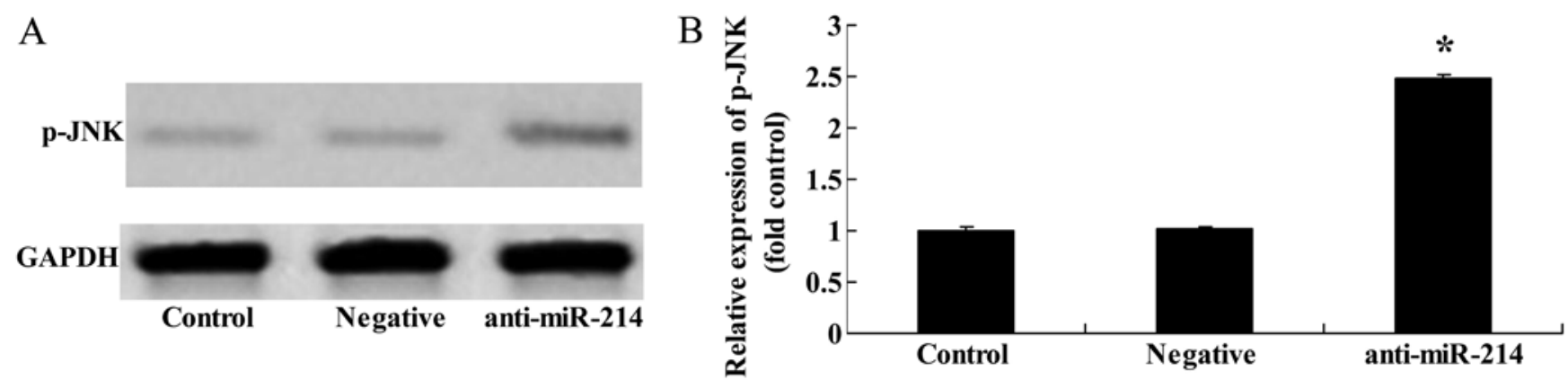

Figure 9. Downregulation of miR-214 increases p-c-Jun N-terminal kinase (JNK) protein expression in bone marrow-derived mesenchymal stem cells (BMSCs). Downregulation of miR-214 suppressed p-JNK protein expression as shown by (A) western blot analysis and (B) statistical analysis of p-JNK protein expression in BMSCs. Control, control group; negative, negative infection group; anti-miR-124, anti-miR-124 infection group. " $\mathrm{p}<0.01$ compared with the control group.

following the overexpression of miR-214, we treated the BMSCs with JNK inhibitor during the course of osteogenic differentiation following the overexpression of miR-214. The use of the JNK inhibitor, SP600125, significantly suppressed 

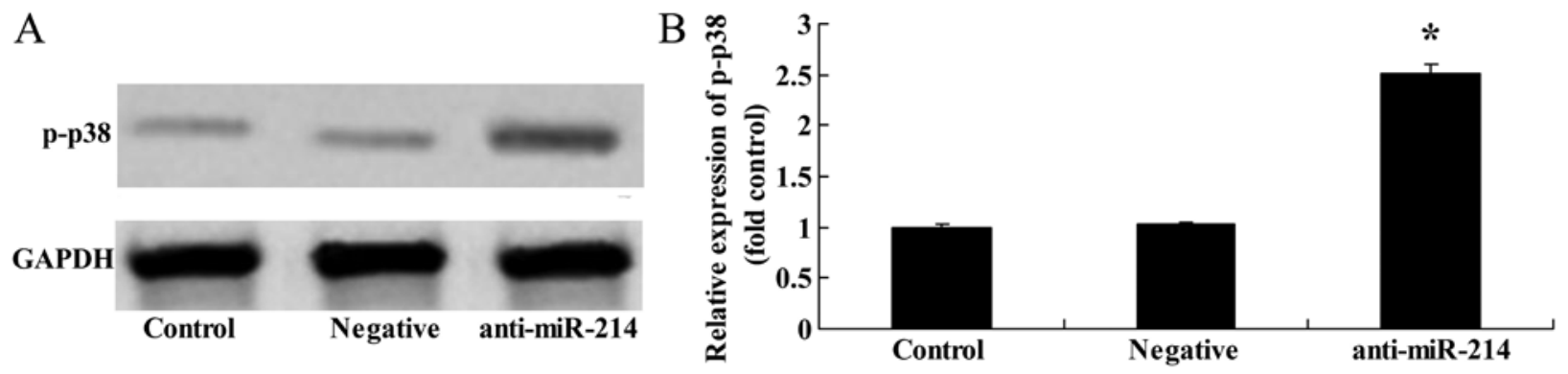

Figure 10. Downregulation of miR-214 increases p-p38 protein expression in bone marrow-derived mesenchymal stem cells (BMSCs). Downregulation of miR-214 suppressed p-p38 protein expression as shown by (A) western blot analysis and (B) statistical analysis of p-p38 protein expression in BMSCs. Control, control group; negative, negative infection group; anti-miR-124, anti-miR-124 infection group. "p $<0.01$ compared with the control group.
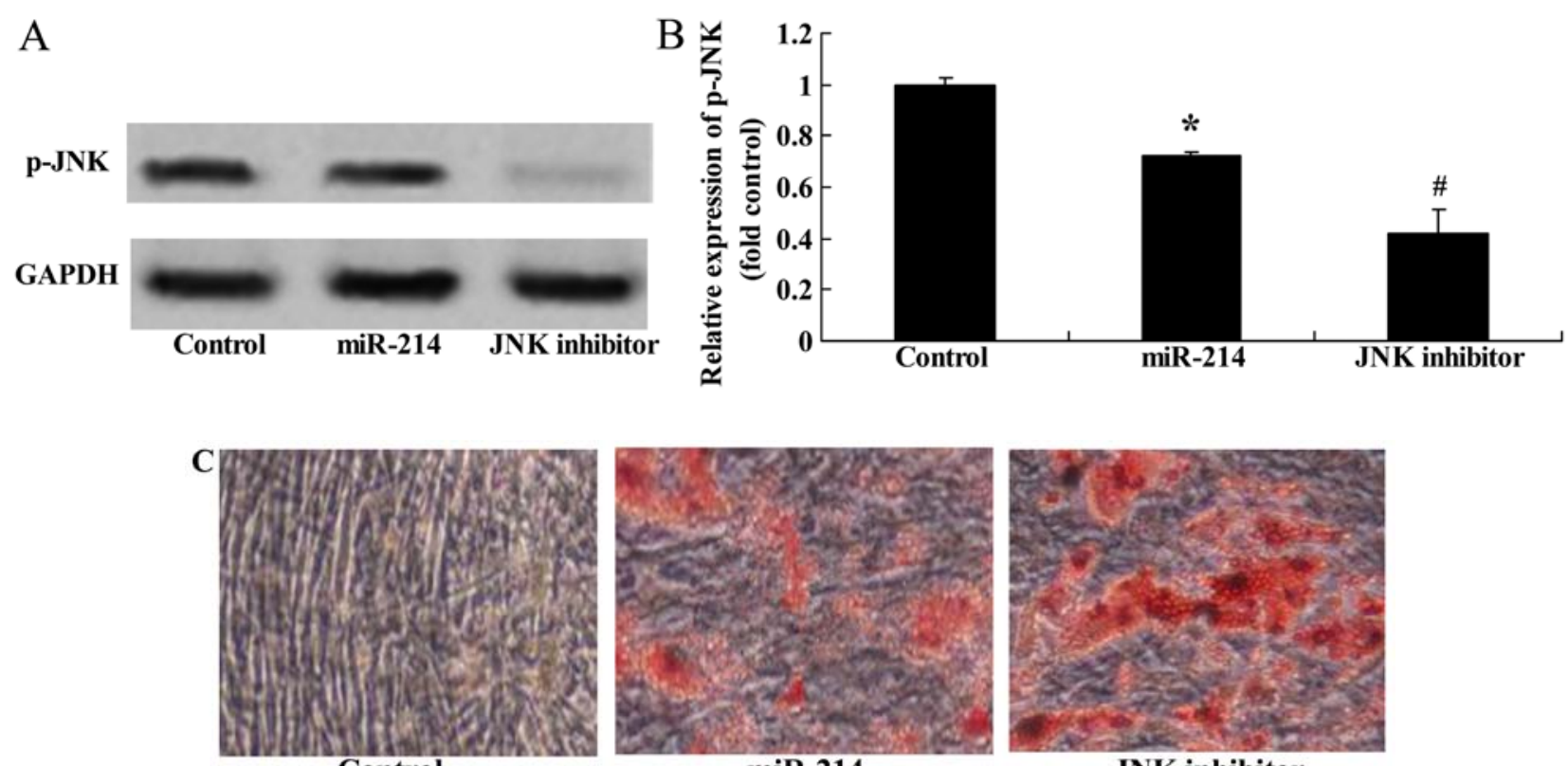

Figure 11. Inhibition of c-Jun N-terminal kinase (JNK) expression enhances the promoting effects of miR-214 on adipocyte differentiation and decreases p-JNK protein expression in bone marrow-derived mesenchymal stem cells (BMSCs) following the overexpression of miR-214. p-JNK protein expression was even more significantly suppressed by treatment of the cells with JNK inhibitor as shown by (A) western blot analysis and (B) statistical analysis of p-JNK protein expression. (C) Inhibition of JNK expression enhanced the adipocyte differentiation of BMSCs, shown by Oil Red O staining; magnification, x400. Control, control group; miR-124, miR-124 infection group; JNK inhibitor, JNK inhibitor (SP600125) group. " $\mathrm{p}<0.01$ compared with the control group; "compared with the miR-124 infection group.

the protein expression of p-JNK (Fig. 11A and B) and enhanced the promoting effects of miR-214 on the adipocyte differentiation of BMSCs (Fig. 11C).

Effect of the inhibition of JNK expression on ALP activity, and Col I, OCN and OPN gene expression in BMSCs following the overexpression of $m i R-214$. We then examined the effects of the inhibition of JNK expression on ALP activity, and Col I, OCN and OPN gene expression in BMSCs following the overexpression of miR-214. The use of the JNK inhibitor, SP600125, significantly inhibited ALP activity, and decreased Col I, OCN and OPN gene expression in the BMSCs following the overexpression of miR-214 (Fig. 12).

Effect of the inhibition of p38 expression on the adipocyte differentiation of BMSCs following the overexpression of $m i R-214$. To further assess the significance of the inhibition of p38 expression on the adipocyte differentiation of BMSCs following the overexpression of miR-214, we treated the
BMSCs with p38 inhibitor during the course of differentiation following the overexpression of miR-214. The use of the p38 inhibitor, SB202190, significantly suppressed the protein expression of p-p38 (Fig. 13A and B) and enhanced the promoting effect of miR-214 on the adipocyte differentiation of BMSCs (Fig. 13C).

Effect of the inhibition of of 338 expression on ALP activity, and Col I, OCN and OPN gene expression in BMSCs following the overexpression of $m i R-214$. Finally, we examined the effect of the inhibition of $\mathrm{p} 38$ expression on ALP activity, and Col I, OCN and OPN gene expression in BMSCs following the overexpression of miR-214. The use of the $\mathrm{p} 38$ inhibitor, SB202190, significantly inhibited ALP activity, and decreased Col I, OCN and OPN gene expression in the BMSCs following the overexpression of miR-214 (Fig. 14). Thee findings indicated that the use of the p38 inhibitor enhanced the inhibitory effects of miR-214 on the osteogenic differentiation of BMSCs. 

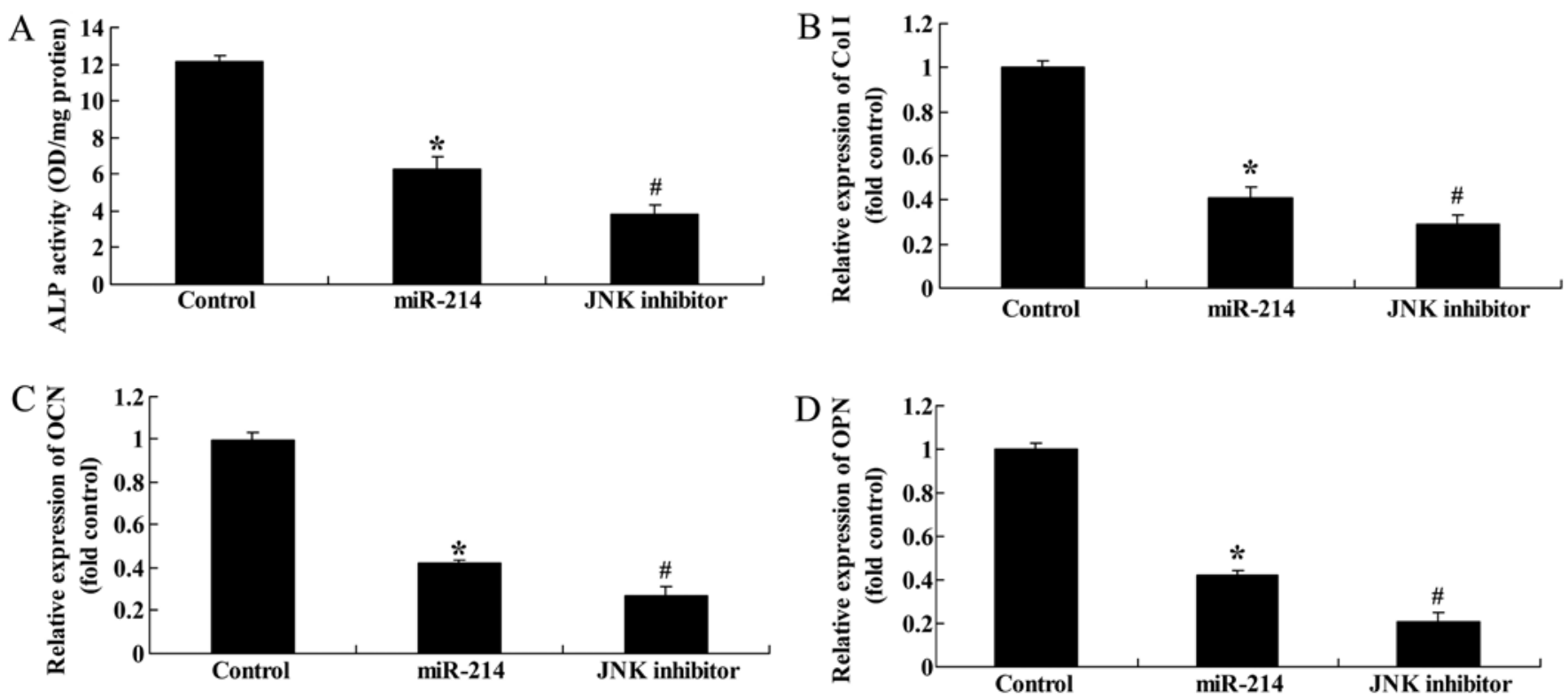

Figure 12. Inhibition of c-Jun N-terminal kinase (JNK) expression enhances alkaline phosphatase (ALP) activity, and increases collagen type I (Col I), osteocalcin $(\mathrm{OCN})$ and osteopontin (OPN) gene expression in bone marrow-derived mesenchymal stem cells (BMSCs) following the overexpression of miR-214. Inhibition of JNK expression increased (A) ALP activity, and (B) Col I, (C) OCN and (D) OPN gene expression in BMSCs. Control, control group; miR-124,

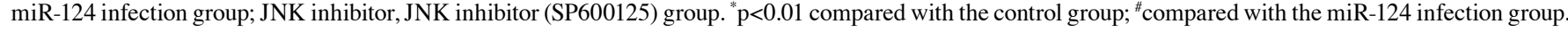
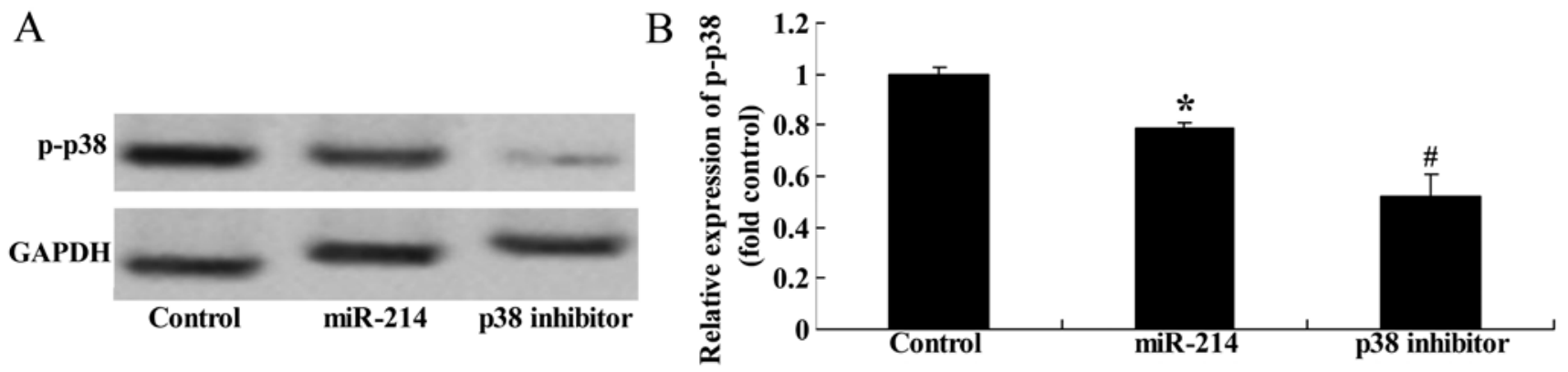

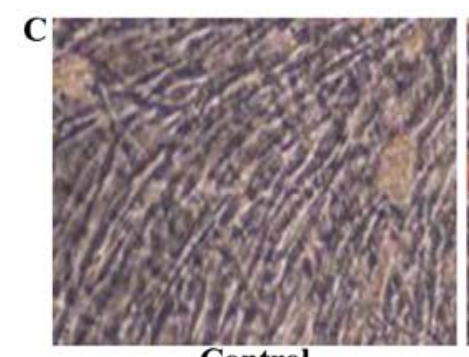

Control

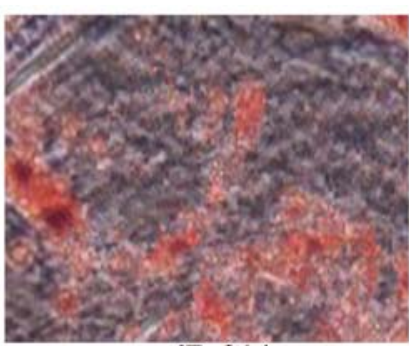

miR-214

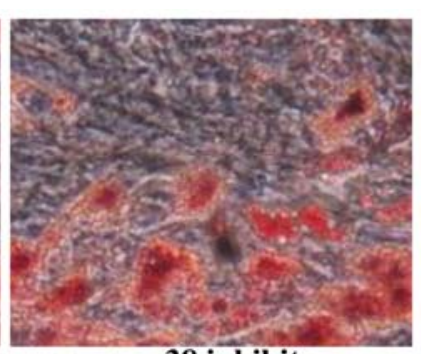

p38 inhibitor

Figure 13. Inhibition of $\mathrm{p} 38$ expression enhances the promoting effect of miR-214 on the adipocyte differentiation of bone marrow-derived mesenchymal stem cells (BMSCs) following the overexpression of miR-214. p-p38 protein expression decreased following treatment with p38 inhibitor, as shown by (A) western blot analysis, and (B) statistical analysis of p-p38 protein expression. (C) Inhibition of p38 expression promoted the adipocyte differentiation of BMSCs, shown by Oil Red O staining; magnification, x400. Control, control group; miR-124, miR-124 infection group; p38 inhibitor, p38 inhibitor (SB202190) group. "p<0.01 compared with the control group; ${ }^{\#}$ compared with the miR-124 infection group.

\section{Discussion}

As adult stem cells from mesoblast, BMSCs do not only differentiate into adult cells from mesoblasts, including osteocytes, chondrocytes and adipocytes under the function of an appropriate inducer for culture in vitro, but can also be transdifferentiated into nerve cells from the neuroderm (4). However, whether BMSCs can differentiate into nerve cells has become a top of extensive investigation. The differentiation mechanisms can be studied from different angles, which can be summarized as: cellular mechanisms of toxicity, cellular fusion mechanism and transdifferentiation (5). Cellular toxicity refers to certain oxidative stress reaction and results in tearing of the cytoskeleton of BMSCs, shrinking of the cytoplasm, and cell shrinking 

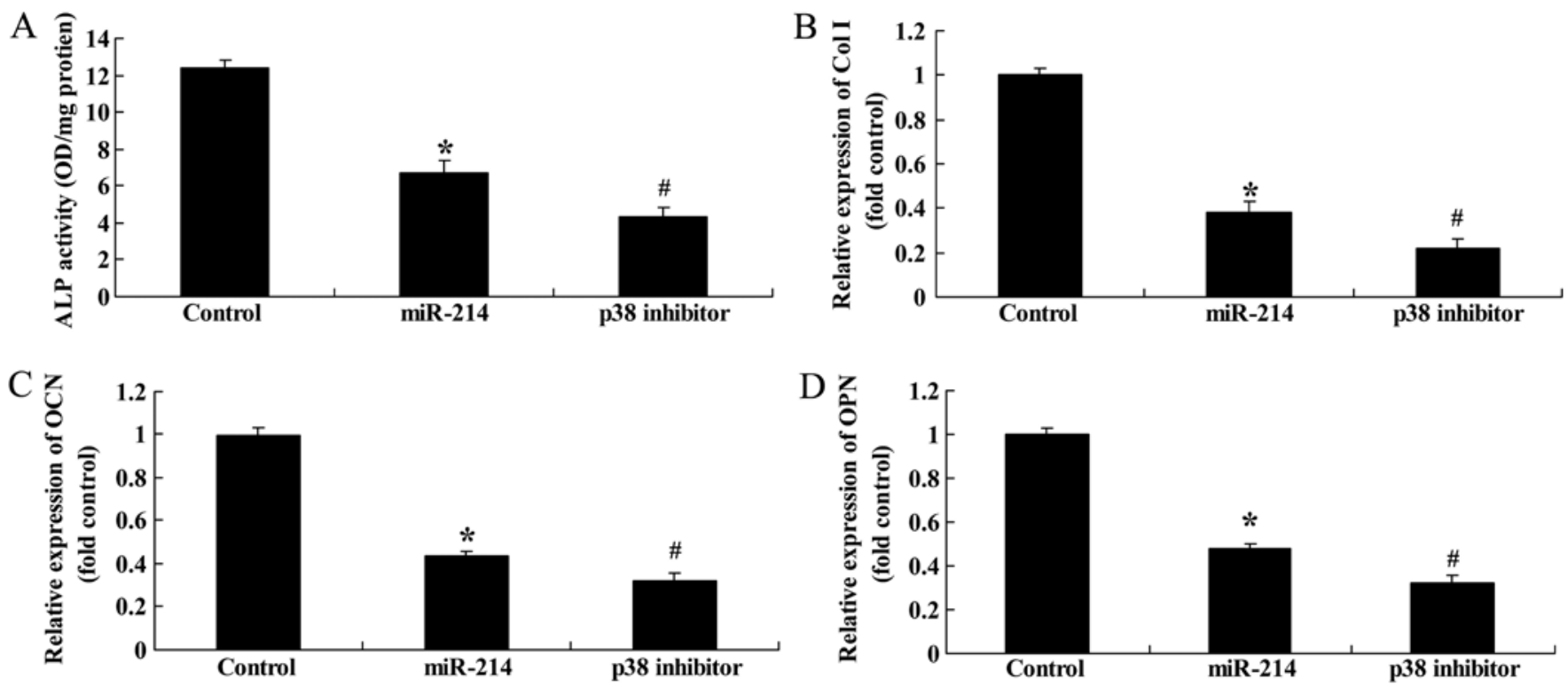

Figure 14. Inhibition of p38 expression decreases alkaline phosphatase (ALP) activity and collagen type I (Col I), osteocalcin (OCN) and osteopontin (OPN) gene expression in bone marrow-derived mesenchymal stem cells (BMSCs) following the overexpression of miR-214. Inhibition of p38 expression decreased (A) ALP activity, and (B) Col I, (C) OCN and (D) OPN gene expression in BMSCs. Control, control group; miR-124, miR-124 infection group; p38 inhibitor, p38 inhibitor (SB202190) group. * $\mathrm{p}<0.01$ compared with the control group; "compared with the miR-124 infection group.

as regards morphology (12). Cytoskeletal proteins are extended to form vimineous and prominent structures similar to nerve cells (13). Changes in biology and molecular remodeling are derived from the toxicity of the inducer on the BMSCs. Thus, they do not really differentiate into neuronal-like cells (13). Cellular fusion mechanism refers to when BMSCs and nerve cells are cultured together, and thus two cells become fused, resulting in gene fusion and the regulation of the gene expression of BMSCs, making the morphology and functions similar to those of nerve cells (14). In this study, the overexpression of miR-214 suppressed osteogenic differentiation and the downregulation of miR-214 activated thez osteogenic differentiation of BMSCs.

ALP is a type of exoenzyme of osteoblasts and a marker of osteogenic differentiation at the early stage. Its expressive activity is an obvious characteristic of osteoblast differentiation (15). ALP can be used for identifying the degree of osteogenic differentiation at the early stage for BMSCs (16). During the later period of differentiation, OCN (also known as bone $\gamma$-carboxylic glutamic acid containing protein) is a type of protein composed and secreted by osteoblasts. Through the gene expression level of OCN, we can identify osteoblasts, particularly at the active stage of newly-formed osteoblasts. This study demonstrated that the overexpression of miR-214 suppressed ALP activity, and Col I, OCN and OPN gene expression. The downregulation of miR-214 enhanced ALP activity, and increased Col I, OCN and OPN gene expression in the BMSCs. Ramazzotti et al (17) indicated that the inhibition of miR-214 enhanced ALP activity and osterix expression in C2C12 cells.

Integrin is a heterosexual cell adhesion molecule. In combination with the extracellular matrix, they form adhesion plaque compounds with other molecules (FAK, talin, vinculi and paxillin) to mediate interactions between cells and the extracellular matrix, as well as mutual communications between intracellular signals and extracellular cells, including force signals. Adhesion plaque is an important signaling molecular compound connecting with intracellular and extracellular environments. FAK is a star molecule in adhesion plaque. Studies have shown that FAK plays an important regulatory role in related gene expression and the fate of cell differentiation. The study by Torsoni et al (18) demonstrated that mechanical stretching can regulate Tyr phosphorylation at FAK 397 sites through integrin. Moreover, FAK phosphorylation is an important regulatory signaling molecule of $\beta-\mathrm{MHC}$ induced by mechanical stretching. Moreover, FAK is also an important induction molecule of force signals relating to cellular feeling matrix hardness. It can induce different matrix hardness and adjust BMSCs induced by matrix hardness to differentiate into osteoblast. FAK thus plays a decisive role in the process of force signal transduction and the induction of stem cell differentiation. In the present study, the upregulation of miR-214 suppressed FAK protein expression and the downregulation of miR-214 activated FAK protein expression in BMSCs. Yang et al suggested that miR-214 inhibits osteogenic differentiation by targeting the FGFR1/FGF signaling pathway in mesenchymal stem cells (19).

MAPK is a type of serine/threonine protein kinase, and is distributed in cell plasma and is equipped with the double phosphorylation of serine and tyrosine. The MAPK pathway in eukaryocytes is extremely abundant (10). The pathway participates in cellular activities through interaction, such as cellular genetic expression, mitosis, metabolism, autonomic activities, proliferation, apoptosis, or even differentiation. MAPK is a type of serine/threonine protein kinase in cells, mainly including ERKs, JNKs and p38 MAPK $(8,10)$. Generally speaking, the ERK signal pathway is activated by mitogens, such as the growth factor, IGFs and FGFs (20). On 
the other hand, the JNK signaling pathway and p38 MAPK signaling pathway are mainly activated through bone morphogenetic proteins (BMPs) and oxidative stress. Three signaling pathways of MAPKs participate in the proliferation and differentiation of osteoblasts (10). In a previous study, following the co-culture of BMSCs, it was found that the phosphorylation level of p38 MAPK and ERK1/2 protein kinase increased (20). In the present study, we found that the upregulation of miR-214 suppressed p-JNK and p-p38 protein expression, and the downregulation of miR-214 increased p-JNK and p-p38 protein expression in BMSCs.

The JNK/p38 MAPK signaling pathway exist in all mammals, and participate in and regulate multiple biological reactions (21). After MEK identifies JNK/p38 MAPK selectively, the conservative threonine-glycine-tyrosine sequence in JNK/p38 MAPK subtype is phosphorylated (22). The corresponding subtype of JNK/p38 MAPK is activated, so as to activate JNK/p38 MAPK signaling. JNK/p38 MAPK in rats provides a safeguard for the normal development of the skeleton (22). If coding gene in any MAPK signal transduction pathway is deficient, such as MKK3, MKK6, p38a or p38b, this results in the reduction of bone mass and osteoblast differentiation defects (23). In osteoblasts, Transforming growth factor- $\beta$ (TGF- $\beta$ )-activated kinase 1 (TAK1) TGF- $\beta$ is the key site of activating upstream JNK/p38 MAPK (24). In this study, we found that the inhibition of JNK/p38 MAPK enhanced the inhibitory effect of miR-214 on osteogenic differentiation and inhibited ALP activity and Col I, OCN and OPN gene expression in BMSCs. This result indicated that JNK/p38 MAPK plays a role in the effects of miR-214 on the osteogenic differentiation of BMSCs.

In the present study, we demonstrate that miR-214 inhibits the osteogenic differentiation of BMSCs, and results in the suppression of the JNK and p38 pathways. Importantly, our data indicate that miR-214 may have potential for use in chronic inflammatory bone diseases.

\section{References}

1. Huang S, Xu L, Zhang Y, Sun Y and Li G: Systemic and local administration of allogeneic bone marrow-derived mesenchymal stem cells promotes fracture healing in rats. Cell Transplant 24: 2643-2655, 2015.

2. Carvalho A, Pelaez-Vargas A, Hansford DJ, Fernandes MH and Monteiro FJ: Effects of line and pillar array microengineered $\mathrm{SiO}_{2}$ thin films on the osteogenic differentiation of human bone marrow-derived mesenchymal stem cells. Langmuir 32: 1091-1100, 2016.

3. Wang W, Du Z, Yan J, Ma D, Shi M, Zhang M, Peng C and Li H: Mesenchymal stem cells promote liver regeneration and prolong survival in small-for-size liver grafts: Involvement of C-Jun N-terminal kinase, cyclin D1, and NF-кB. PLoS One 9: e112532, 2014.

4. Baniwal SK, Shah PK, Shi Y, Haduong JH, Declerck YA, Gabet Y and Frenkel B: Runx2 promotes both osteoblastogenesis and novel osteoclastogenic signals in ST2 mesenchymal progenitor cells. Osteoporos Int 23: 1399-1413, 2012.

5. Xu JF, Yang GH, Pan XH, Zhang SJ, Zhao C, Qiu BS, Gu HF, Hong JF, Cao L, Chen Y, et al: Altered microRNA expression profile in exosomes during osteogenic differentiation of human bone marrow-derived mesenchymal stem cells. PLoS One 9: e114627, 2014.

6. Skårn M, Namløs HM, Noordhuis P, Wang MY, Meza-Zepeda LA and Myklebost O: Adipocyte differentiation of human bone marrow-derived stromal cells is modulated by microRNA-155, microRNA-221, and microRNA-222. Stem Cells Dev 21: 873-883, 2012.
7. Qadir AS, Um S, Lee H, Baek K, Seo BM, Lee G, Kim GS, Woo KM, Ryoo HM and Baek JH: miR-124 negatively regulates osteogenic differentiation and in vivo bone formation of mesenchymal stem cells. J Cell Biochem 116: 730-742, 2015.

8. Dai Z, Li Y, Quarles LD, Song T, Pan W, Zhou H and Xiao Z: Resveratrol enhances proliferation and osteoblastic differentiation in human mesenchymal stem cells via ER-dependent ERK1/2 activation. Phytomedicine 14: 806-814, 2007.

9. Liao QC, Xiao ZS, Qin YF and Zhou HH: Genistein stimulates osteoblastic differentiation via p38 MAPK-Cbfa1 pathway in bone marrow culture. Acta Pharmacol Sin 28: 1597-1602, 2007.

10. Wu X, Chen S, Orlando SA, Yuan J, Kim ET, Munugalavadla V, Mali RS, Kapur R and Yang FC: p85alpha regulates osteoblast differentiation by cross-talking with the MAPK pathway. J Biol Chem 286: 13512-13521, 2011.

11. Soleimani M and Nadri S: A protocol for isolation and culture of mesenchymal stem cells from mouse bone marrow. Nat Protoc 4: 102-106, 2009.

12. Laitinen A, Oja S, Kilpinen L, Kaartinen T, Möller J, Laitinen S, Korhonen $\mathrm{M}$ and Nystedt J: A robust and reproducible animal serum-free culture method for clinical-grade bone marrowderived mesenchymal stromal cells. Cytotechnology 68: 891-906, 2016.

13. Da Silva JS and Hare JM: Cell-based therapies for myocardial repair: Emerging role for bone marrow-derived mesenchymal stem cells (MSCs) in the treatment of the chronically injured heart. Methods Mol Biol 1037: 145-163, 2013.

14. Lv S, Liu G, Sun A, Wang J, Cheng J, Wang W, Liu X, Nie H and Guan G: Mesenchymal stem cells ameliorate diabetic glomerular fibrosis in vivo and in vitro by inhibiting TGF- $\beta$ signalling via secretion of bone morphogenetic protein 7. Diab Vasc Dis Res 11: 251-261, 2014.

15. Tao K, Xiao D, Weng J, Xiong A, Kang B and Zeng H: Berberine promotes bone marrow-derived mesenchymal stem cells osteogenic differentiation via canonical Wnt//-catenin signaling pathway. Toxicol Lett 240: 68-80, 2016.

16. Kim SE, Jeon O, Lee JB, Bae MS, Chun HJ, Moon SH and Kwon IK: Enhancement of ectopic bone formation by bone morphogenetic protein-2 delivery using heparin-conjugated PLGA nanoparticles with transplantation of bone marrowderived mesenchymal stem cells. J Biomed Sci 15: 771-777, 2008.

17. Ramazzotti G, Bavelloni A, Blalock W, Piazzi M, Cocco L and Faenza I: BMP-2 Induced expression of PLC $\beta 1$ that is a positive regulator of osteoblast differentiation. J Cell Physiol 231: 623-629, 2016.

18. Torsoni AS, Constancio SS, Nadruz W Jr, Hanks SK and Franchini KG: Focal adhesion kinase is activated and mediates the early hypertrophic response to stretch in cardiac myocytes. Circ Res 93: 140-147, 2003.

19. Yang L, Ge D, Cao X, Ge Y, Chen H, Wang W and Zhang H: miR-214 attenuates osteogenic differentiation of mesenchymal stem cells via targeting FGFR1. Cell Physiol Biochem 38: 809-820, 2016.

20. Martinez-Lopez N and Singh R: ATGs: Scaffolds for MAPK/ERK signaling. Autophagy 10: 535-537, 2014.

21. Dong J, Wang J, He Y, Li C, Zhou A, Cui J, Xu W, Zhong L, Yin Y, Zhang X and Wang H: GHIP in Streptococcus pneumoniae is involved in antibacterial resistance and elicits a strong innate immune response through TLR2 and JNK/p38 MAPK. FEBS J 281: 3803-3815, 2014

22. Lee CM, Onésime D, Reddy CD, Dhanasekaran N and Reddy EP: JLP: A scaffolding protein that tethers JNK/p38 MAPK signaling modules and transcription factors. Proc Natl Acad Sci USA 99: 14189-14194, 2002.

23. Pang HY, Liu G and Liu GT: Compound FLZ inhibits lipopolysaccharide-induced inflammatory effects via downregulation of the TAK-IKK and TAK-JNK/p38 MAPK pathways in RAW264.7 macrophages. Acta Pharmacol Sin 30: 209-218, 2009.

24. Kuroyanagi G, Otsuka T, Yamamoto N, MatsushimaNishiwaki R, Kozawa O and Tokuda H: Resveratrol suppresses TGF- $\beta$-induced VEGF synthesis in osteoblasts: Inhibition of the p44/p42 MAPKs and SAPK/JNK pathways. Exp Ther Med 9: 2303-2310, 2015 . 